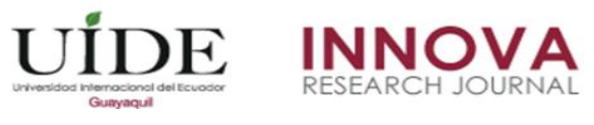

INNOVA Research Journal, ISSN 2477-9024

(Diciembre, 2018). Vol. 3, No.12 pp. 159-166

DOI: https://doi.org/10.33890/innova.v3.n12.2018.842

URL: http://revistas.uide.edu.ec/index.php/innova/index

Correo: innova@uide.edu.ec

\title{
La economía popular y solidaria de la producción de alimentos derivados del pescado del cantón Santa Rosa
}

\section{The popular and solidarity economy of the production of food derived from fish from the Santa Rosa canton}

Tatiana Ximena Sánchez Quezada

Iván Gordillo Quizhpe

Rody Huanca Huanca

Universidad Técnica de Machala, Ecuador

Autora para correspondencia: tsanchez@utmachala.edu.ec

Fecha de recepción: 08 de junio de 2018 - Fecha de aceptación: 01 de diciembre de 2018

Resumen: La investigación se fundamenta en principios y enfoques teóricos de Economía Popular y Solidaria a través de un análisis de familias emprendedoras dedicadas a la preparación de alimentos derivados del pescado del cantón Santa Rosa. El objetivo demuestra teórica y estadísticamente que las actividades populares nacen desde factores de oportunidad e iniciativas entre familiares y amigos, progresivamente se constituyen en alternativas viables que promueven rentabilidad, así como ampliación de calidad de vida y generación de fuentes de trabajo solidario. La metodología se basó en un enfoque mixto; de nivel exploratorio y descriptivo. En la recolección de datos se aplicaron entrevistas, observación de campo y encuestas estructuradas. El universo constituyó el total de familias de la "nueva economía social". Los resultados muestran que las familias analizadas tienen un alto nivel de compromiso con el emprendimiento, siendo la única fuente de ingresos que poseen con márgenes de rentabilidad y bajo nivel de inversión, la relación costo beneficio es favorable en contextos familiares bajo principios de reciprocidad y complementariedad articulada a la economía local. El estudio establece que los factores que fundamentan la actividad productiva se basan en acuerdos de identidad cultural, mecanismos de reconocimiento, sostenibilidad ambiental, promoviendo principios de solidaridad e integralidad.

Palabras Claves: economía popular y solidaria; emprendimiento; familias emprendedoras; oportunidad

Abstract: The research is based on principles and theoretical approaches of Popular and Solidarity Economy through an analysis of entrepreneur families dedicated to the preparation of foods derived from fish from the Santa Rosa canton. The objective demonstrates theoretically and statistically that popular activities are born from opportunity factors and initiatives between family and friends, progressively constitute viable alternatives that promote profitability, as well as an increase in the quality of life and the generation of sources of solidary work. The methodology was based on a mixed approach; of exploratory and descriptive level. In the data collection, interviews, field observation and structured surveys were applied. The universe constituted the total of families of the "new social economy". The results show that the families analyzed have a high level of commitment to entrepreneurship, being the only source of income they have with profitability margins and low level of investment, the cost-benefit ratio is favorable in family contexts under the principles of reciprocity and complementarity articulated to the local economy. The study establishes that the factors that base the productive activity are based on agreements of cultural identity, mechanisms of recognition, environmental sustainability, promoting principles of solidarity and integrality.

Key words: popular and solidarity economy; entrepreneurship; entrepreneurial families; opportunity 


\section{Introducción}

Es oportuno preguntarse, cual es el contexto y a que responde la economía popular y solidaria, ante lo cual Coraggio (2011, pág. 45), expresa, que aparece desde muchos matices y niveles macro, estructurales, incluso globales, frente a la supremacía de la economía de mercado y la acumulación lucrativa, teniendo factores preponderantes, la exclusión social, la brechas de desigualdad, pobreza y las crisis de identidad; pero también responde a la deficiente capacidad del Estado, con la institucionalización de las iniciativas asociativas económicas sociales; incluso surge desde matrices ecológicos y culturales ancestrales, con propósitos de recuperación y revalorización de lo diverso, la complementariedad del ser humano con los "derechos de la naturaleza", frente a lo antropogénico y la crisis ambiental a nivel global y local. Marco sobre el cual se desprenden las tendencias, enfoques, teorías explicativas y fundantes de la economía popular solidaria. Teniendo como factor común, la prevalencia del trabajo sobre el capital, estableciéndose entre el Estado, la sociedad y el mercado global, las lógicas de la reciprocidad (Obando, 2009).

La economía popular y solidaria en el caso de Ecuador, la "contempla como política pública" y está establecida dentro del ordenamiento jurídico, al considerarla como la "forma de organización económica, donde sus integrantes organizan y desarrollan procesos de producción, intercambio, comercialización, financiamiento y consumo de bienes y servicios, para satisfacer necesidades y generar ingresos, en armonía con la naturaleza" y orientada a la concesión de los derechos y los objetivos del buen vivir, donde "prevalece el ser humano sobre el capital". (LOEPS, 2011). Con lo cual se afirma, que la economía popular y solidaria, reconoce y reivindica algunos principios de la cual se nutre y se basan en los factores C: cooperación, comunitarismo, confianza colaboración, compañerismo. En esta dinámica del proceso productivo, se evidencia que no prevalece el lucro y la rentabilidad, más bien está primero el ser humano que el capital.

En este sentido, se define a la economía popular solidaria, como el conjunto de capacidades y recursos que se articulan con carácter social, sin fines de lucro, y fundadas en valores y factores sociales e identitarios de orden cultural en la "realización de actividades de producción, distribución, circulación, financiamiento y consumo, de las unidades domésticas (familiares y comunales), y las organizaciones específicas (...) con el objetivo de reproducir la vida y fuerza de trabajo en las mejores condiciones posibles", incluso sin costos más que los principios corporativos de reciprocidad y soportando las presiones culturales de la económica del capital. (Ramón, Arteaga - Macías, 2017, p, 4).

Así, los emprendedores asociativos del Cantón Santa Rosa que transforman los derivados del pescado, se evidencia que promueven un tipo de economía con perspectiva social, basado en el trabajo y capital familiar, regentado en pactos de crecimiento solidario. Lo que implica que la economía social, enfocada desde cualquier análisis, es opuesta y crítica a la economía de mercado y acumulación de capital, incluso ajena al Estado. En este contexto, se propuso la pregunta de investigación: ¿Los emprendimientos asociativos e individuales de la economía popular y solidaria sostienen los ingresos familiares y dinamizan la economía local? 
La respuesta se confirma a modo de hipótesis global con los resultados del estudio que demuestra con efectividad, que las economías sociales, en el caso de los procesadores directos de alimentos del derivados de pescado de Santa Rosa, se fundan por iniciativas de oportunidad y la necesidad de sobrevivencia, dinamizan los factores productivos, en base al trabajo y capital social familiar, hacen uso de limitada tecnología, el financiamiento lo obtienen por ahorro y préstamo, la gestión y administración se establece en base a la principios de la complementariedad en la que todos los que participan del negocio asociativo "deben apoyar" y los márgenes de utilidad lo capitalizan en bienes muebles e inmuebles, incluso en ahorro que al final se constituyen en patrimonio familiar.

La propuesta metodológica responde a la categorización de variables desde los enfoques cuantitativo y cualitativo, con el apoyo del análisis explotaría - descriptivo, y el proceso de Operacionalización, a través de indicadores que hizo posible la elaboración de la base teórica, formulación de hipótesis de trabajo y el diseño de técnicas e instrumentos como la entrevista y la encuesta. Con las cuales se procedió a la recolección de datos de la población del universo sujeto de estudio, el procesamiento, la interpretación y discusión. Cumpliéndose de esta forma el ciclo epistemológico del método científico y metodológico de la investigación.

\section{Materiales y Métodos}

El tipo de metodología que se utiliza en una investigación depende del tema y el problema que se aborde

"La investigación científica es un modo específico de plantear y resolver problemas de conocimiento acerca de la realidad. La modalidad científica de indagación del mundo se caracteriza tanto por el modo en que el investigador se interroga sobre los fenómenos, como por la forma en que interroga a los fenómenos en su afán de encontrar respuestas a los dilemas que se plantea" (Yuni \& Urbina, 2014)

El diseño del instrumento responde a las variables y su proceso de Operacionalización a través de categorías e indicadores que hizo posible la elaboración de técnicas e instrumentos de investigación. Adicional se procedió a su validación en poblaciones homogéneas que permita establecer el coeficiente de confiabilidad y que finalmente concluye con la aplicación orientada a recoger los datos de la población sujeto de estudio.

En la investigación se dispone de varios tipos de instrumentos para medir las variables de interés, uno de ellos son los cuestionarios según (Hernández, 2014).

La población de estudio comprende el total de emprendedores asociativos asentados en el cantón Santa Rosa de la provincia de El Oro - Ecuador, que equivale al conjunto del universo, lo que no dio lugar a estimaciones y probabilidades estadísticas, facilitando la recopilación de información de datos con la aplicación de encuestas a los actores in situ.

\section{Resultados y Discusión}

De acuerdo a las encuestas realizadas en el cantón Santa Rosa se pudo identificar que el rango de edad con mayor porcentaje 52\% dedicado a esta actividad en el Cantón es de 40 a 50 
años, con experiencia de más de diez años en el negocio y el género que predomina es el masculino con el $71 \%$.

La motivación es un aspecto relevante en las diversas áreas de la vida, entre ellas la educativa y laboral, orienta las acciones y es un elemento central que conduce lo que la persona realiza y hacia qué objetivos se dirige. (Naranjo Pereira, 2017, pág. 135). En la presente investigación el $61 \%$ de los emprendedores aseguran que sus familiares fueron quienes los motivaron a realizar el emprendimiento mientras que el $23 \%$ sostiene que existe automotivación, es decir, encuentran en ellos mismos el interés, impulso y entusiasmo para realizar su actividad comercial y el $16 \%$ de los emprendedores han tenido una motivación de parte de sus amigos. Así mismo se constató que el principal motivo para emprender de los encuestados fue por necesidad con el 68\%, mientras que el 19\% decidió emprender para mejorar ingresos y el 13\% de los emprendedores tomó la oportunidad de iniciar un negocio y generar empleo a sus allegados. El psicólogo David McClelland (1961) afirma que "la necesidad de logro es lo que realmente lleva a un individuo a convertirse en emprendedor" (Marulanda Valencia \& Morales Gualdrón, 2016, pág. 16).

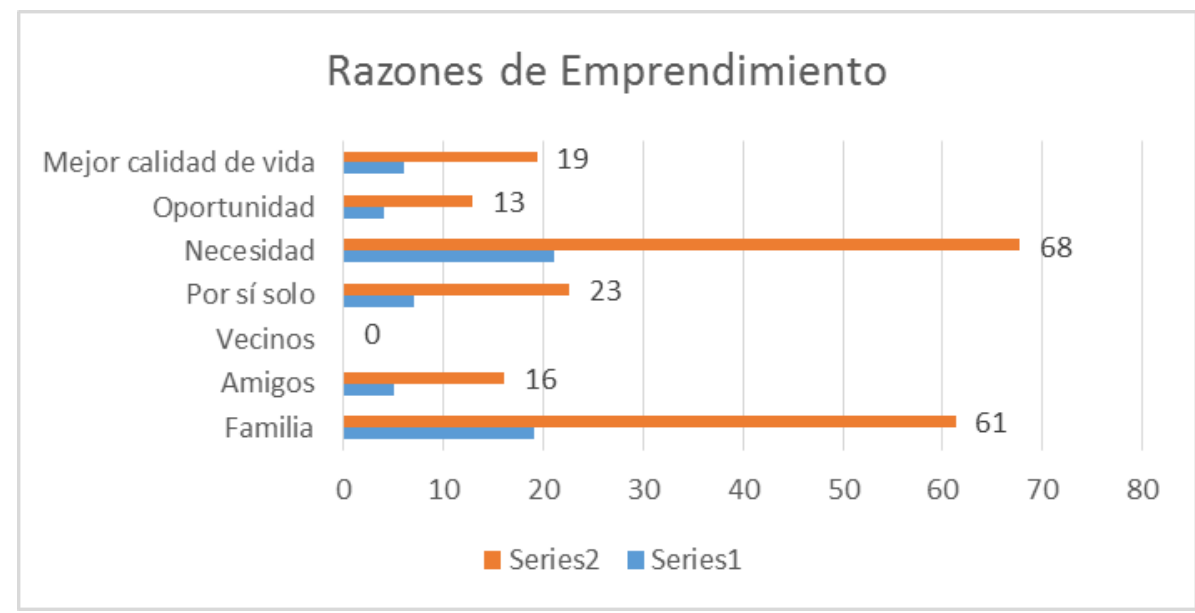

Gráfico 1 Razones de emprendimiento

Fuente: Autores

Así mismo se pudo constatar que las personas involucradas en este tipo de emprendimiento son la familia directamente como el conyugue y los hijos lo cual representa el $65 \%$ y el 35\% restante incluye demás familiares o trabajan solos. De igual forma se verificó que el $55 \%$ de los encuestados contratan máximo hasta dos personas adicionales para su negocio y en su mayoría para atención al cliente debido a que "no se puede obviar que en los años recientes los clientes han ido evolucionando y son ahora más demandantes, más conocedores, más sofisticados y están más deseosos de tomar sus propias decisiones" (Martínez Delgado \& Lauzardo Rico, 2016, pág. 50). Por lo tanto el $84 \%$ de los emprendedores asegura que el personal extra en su negocio en netamente para atención al cliente, el 10\% contrata personal especializado en la preparación de ceviche y el $6 \%$ restante para actividades de limpieza. 


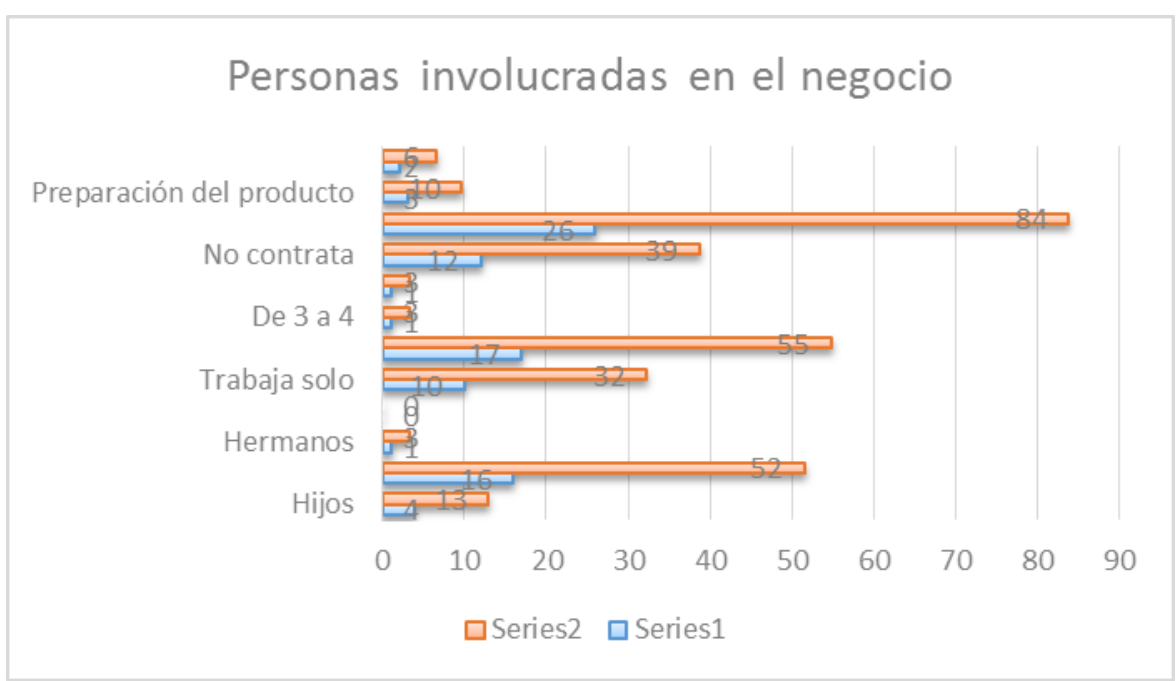

Gráfico 2 Personas involucradas en el negocio

Fuente: Autores

Mediante encuestas realizadas a los expendedores de ceviche en la ciudad de Santa Rosa se pudo identificar que el $68 \%$ de los emprendedores asegura que la mañana es el momento que atienden a sus clientes por lo que es el instante en que el marisco está recién preparado y por tradición el ceviche se consume más en las mañanas, por lo tanto atender al cliente en este horario es la estrategia de venta de los emprendedores. De igual manera del tiempo que dedican a esta actividad por día es de aproximadamente de 8 a 10 horas entre la preparación y expendio del producto, procurando brindar a los clientes mejor atención en el servicio que prestan. Cabe destacar que dar una excelente atención al cliente es de vital importancia para incrementar las ganancias Najul Godoy (2011) afirma:

La atención al cliente es una actividad desarrollada por las organizaciones con orientación a satisfacer las necesidades de sus clientes, logrando así incrementar su productividad y ser competitiva. El cliente es el protagonista principal y el factor más importante en el juego de los negocios. (pág. 25)

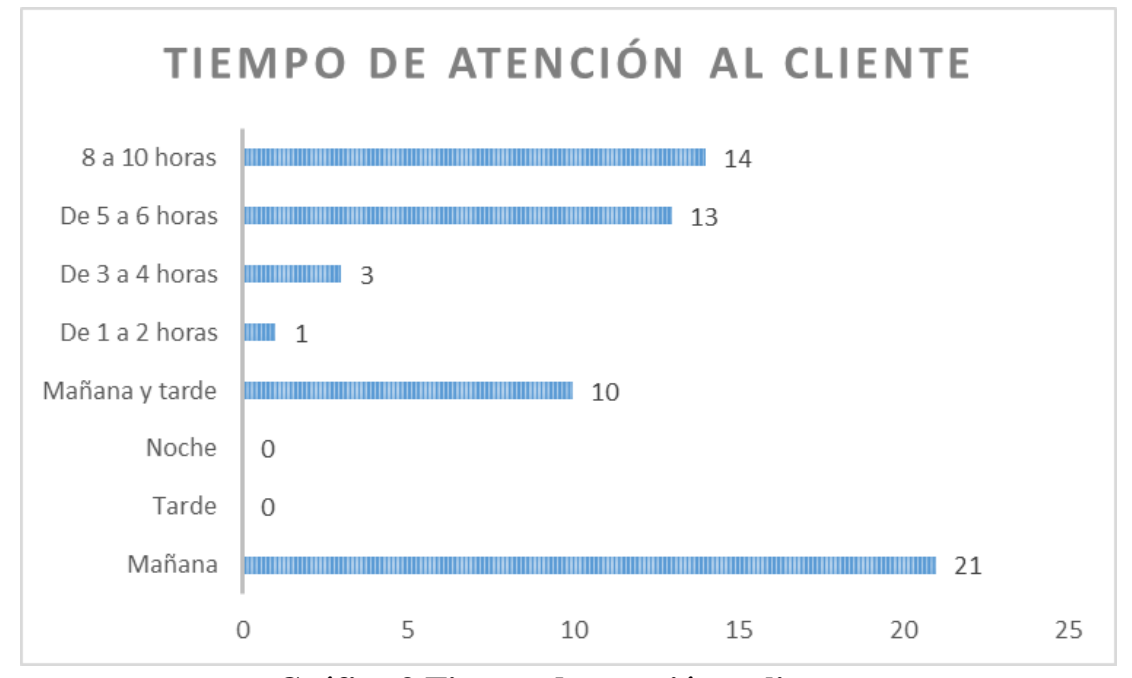

Gráfico 3 Tiempo de atención a clientes Fuente: Autores 
Invertir es un hecho de suma importancia que radica en el uso de los recursos en el sector productivo con la finalidad de lograr beneficios y ganancias, su importancia reside en lograr obtener libertad financiera por lo que $42 \%$ de la población en estudio afirma que reinvertiría su dinero en ampliar su negocio, el 52\% en comprar bienes y el 6\% restante en ahorrar y educación para los hijos. Así mismo se investigó de dónde obtiene el dinero para ampliar el negocio, el $48 \%$ de los emprendedores asevera por medio de préstamos bancarios, por lo que Ríos Bolivar \& Gómez Rodríguez (2015) afirman:

La industria bancaria sirve como el conducto principal a través del cual la inestabilidad puede ser transmitida a otros sectores de la economía mediante: la interrupción del mercado de préstamos interbancarios, la suspensión de los mecanismos de pagos, al reducir la disponibilidad del crédito y al congelar los depósitos. (pág. 40)

El ser humano debe priorizar sus gastos y necesidades personales por lo tanto el $45 \%$ de las personas encuestadas convienen que su prioridad en gastos es la alimentación Carcamo Vargas \& Mena Bastías (2016) asegura "La alimentación sana y la actividad física para prevenir enfermedades son instrumentos decisivos para alcanzar los objetivos personales" (pág. 40). Consecuentemente el $32 \%$ de los emprendedores invierten sus recursos en educación y el $23 \%$ en salud siendo estos indicadores vitales para la supervivencia humana.

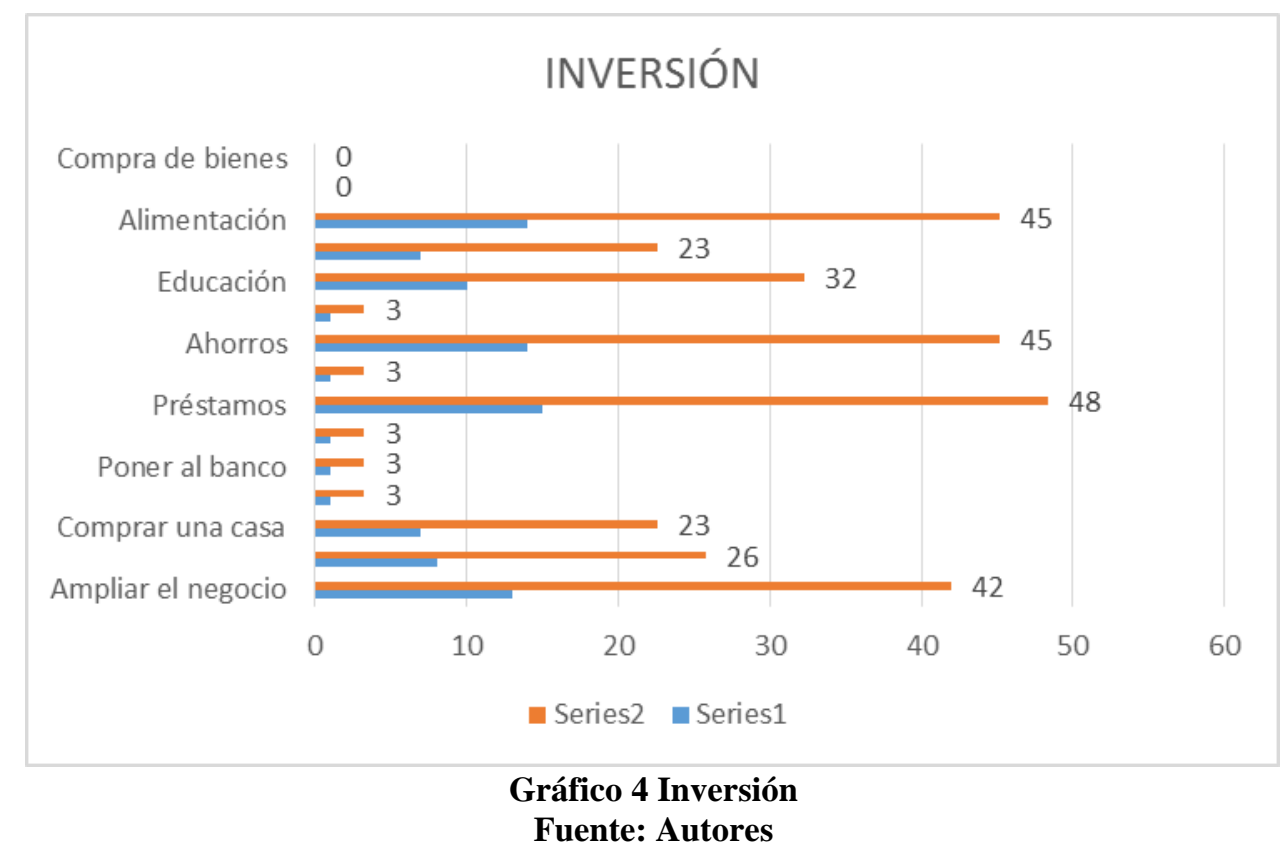

\section{Conclusiones}

Del resultado de las investigaciones y de la misma práctica de la economía social, han surgido enfoques, teorías y categorías impulsadas en Europa, América Latina, y Ecuador, las mismas que requieren re-orientarlas a estudios de la economía popular solidaria de la provincia, en razón de las limitadas investigaciones; y los aportes que brindan la explicación y comprensión de la "otra economía con perspectiva humana, cultural y ecológica"; pero a la vez que resulta critico paradojal, al sostenimiento de la economía global de mercado, y en el otro 
extremo de la polarización participa dentro de un juego de suma cero con mecanismos de exclusión y las limitadas inversiones, las emergentes economías familiares, regentadas por principios de asociatividad sin fines de lucro pero con la búsqueda de la eterna manutención y sobrevivencia de los actores sociales.

Lo extraño de las economías de los emprendedores asociativos de Santa Rosa es que surgen solas, sin academia, ni ciencia, sin el Estado y permanecen solas en las sombras del capital financiero y del mercado, que luego en la incomprensión de la política pública, el Estado participa de las exigencias reguladoras y tributarias que en muchos de los casos desalientan las visiones emprendedoras asociativas.

El factor oportunidad y necesidad es la palanca y la estrategia de enganche que posibilita a la economía social insertarse en el mercado, pero con factores de competitividad en donde prevalece el trabajo y la persona sobre el capital, con los cuales se enfrenta a las economías de mercado y la economía pública.

La distinción de la economía social, con las otras economías, en el proceso productivo, el uso de capital social y del trabajo, la administración del negocio, la inversión y usos de tecnologías, se ubican los acuerdos de mutua reciprocidad, cooperación y solidaridad que van desde lo individual hasta lo familiar e institucional.

\section{Bibliografía}

Asamblea Constituyente (2017). Ley Orgánica de Economía Popular y Solidaria. Publicada en el Registro Oficial No. 16, 16 de junio de 2017. Quito-Ecuador: Registro Oficial, Órgano del Gobierno del Ecuador.

Carcamo Vargas, G., \& Mena Bastías, C. (2016). Alimentación saludable. Horizontes Educacionales, núm. 11, 36-44.

Coraggio, J. (2011). Economía social y solidaria. El trabajo antes que el capital. Ecuador: Ediciones Abya-Yala.

Duarte, T., \& Ruiz Tibana, M. (diciembre de 2009). Emprendimiento, una opción para el desarrollo. Scientia Et Technica, vol. XV, núm. 43, 326-331.

Hernández, R., Fernández, C. \& Baptista, M. (2014). Metodología de la Investigación. (Sexta Edición ed.) México: McGraw-Hill.

López Morín, M. (2015). Capacitación. Conciencia Tecnológica, núm. 27-30, 212-216.

Martínez Delgado, E., \& Lauzardo Rico, J. (mayo - agosto de 2016). El servicio al cliente: una necesidad imperante en la calidad de la industria. Ingeniería Mecánica, vol. 9, núm. 2, 4954. 
Marulanda Valencia, F., \& Morales Gualdrón, S. (julio - diciembre de 2016). Entorno y motivaciones para emprender. Revista Escuela de Administración de Negocios, núm. 81, $12-28$.

Molina Castillo, F., \& Munuera Alemán, J. (octubre - diciembre de 2017). Efectos de la novedad y de la calidad del producto en el resultado a corto y a largo plazo en las empresas innovadoras españolas. Universidad Business Review, núm. 20, 68-83.

Moliner cantos, C. (2013). Calidad de Servicio y Satisfacción del cliente. Revista de Psicología del Trabajo y de las Organizaciones, vol. 17, núm. 2, 233-235.

Najul Godoy, J. (julio-diciembre de 2011). El capital humano en la atención al cliente y la calidad de servicio. Observatorio Laboral Revista Venezolana, 23-25.

Naranjo Pereira, M. (2017). Motivación: perspectivas teóricas y algunas consideraciones de su importancia en el ámbito educativo. Educación, vol. 33, núm. 2, 153-170.

Obando, Diego, (2009), Economía social solidaria ¿en función de un desarrollo alternativo o de un neocapitalismo?, Revista Alteridad, No 88, Quito

Parker Gumucio , C. (julio-diciembre de 2006). La religión y el despertar de los pueblos indígenas en América Latina. Alteridade, 81-90.

Ramón S. Arteaga-Macías, Economía popular y solidaria, República del Ecuador, Número Publicado el 23 de octubre de 2017. Dom. Cien., ISSN: 2477-8818.Vol. 3, núm 4, octubre, 2017, pp. 533-542 http://dx.doi.org/10.23857/dom.cien.pocaip.2017.3.4.oct.533-542 URL:http://dominiodelasciencias.com/ojs/index.php/es/index

Ríos Bolívar, H., \& Gómez Rodríguez, T. (enero - junio de 2015). Competencia, eficiencia y estabilidad financiera en el sector bancario mexicano. Revista Mexicana de Economía y Finanzas. Nueva Época, 39-58.

Servicio de Rentas Internas. (2010). Régimen impositivo simplificado. Recuperado el 14 de 09 de 2018, de http://www.sri.gob.ec/: http://www.sri.gob.ec/web/guest/regimen-impositivosimplificado-rise

Yuni, J. \& Urbano, C. (2014). Técnicas para investigar: recursos metodológicos para la preparación de proyectos de investigación. Argentina: Editorial Brujas. 